\title{
Throughput enhancement using synchronization and three-dimensional resource allocation
}

\author{
Hyuk-Chin Chang ${ }^{*}$ and Saewoong Bahk
}

\begin{abstract}
Emerging multimedia applications require more bandwidth and strict QoS requirements. To meet these in wireless personal area networks, WiMedia multiband-orthogonal frequency division multiplexing (MB-OFDM) has been designed while consuming low-transmission power. In this article, we increase the wireless bandwidth of the standard MB-OFDM scheme three times using device synchronization, and consider resource allocation policies to deal with the increased bandwidth. Then, we apply the proposed allocation policies with some operation rules to support prioritized QoS traffic. Extensive simulations verify that the synchronized MB-OFDM triples the throughput of the standard MB-OFDM, and the considered allocation policies with the considered operation rules run effectively as desired.
\end{abstract}

\section{Introduction}

Wireless technologies have been evolved to support data rates of up to a few hundreds of Mbps for high data rate and QoS services such as voice over internet proto$\mathrm{col}$, internet protocol television, and wireless universal serial bus. Typically, the communication range for such high data rates is within a few tens of meters that covers home or office environments, where wireless personal area network (WPAN) technology provides the communication with high data rate, low-transmission power consumption, and low cost [1]. WiMedia alliance has standardized the PHY and medium access control (MAC) layers for multiband-orthogonal frequency division multiplexing (MB-OFDM) of high data rate WPAN based on ultra wide band (UWB), called ECMA (European Computer Manufacturers Association)-368 [2].

Supporting multimedia traffic with QoS requirements over wireless environments is still an important issue in the resource management. Besides, emerging high-quality video applications such as full high-definition multimedia contents require more bandwidth. The MAC is a key layer to meet tight QoS requirements and achieve high throughput [3-7].

WiMedia MAC has two wireless channel access policies: contention-free distributed reservation protocol (DRP) like time division multiple access (TDMA) and

\footnotetext{
* Correspondence: chc@netlab.snu.ac.kr

School of Electrical Engineering and Computer Science, Seoul National University, Seoul 151-742, Korea
}

contention-based prioritized contention access (PCA) with priorities like IEEE 802.11-2007 [8]. DRP is designed to support QoS for isochronous streams such as multimedia contents [9-11], and PCA to support a random channel access for asynchronous services $[12,13]$. In [3], DRP and PCA are used together to assign I, B, and P frames in H.264/AVC (MPEG-4 Part 10) to the wireless resource. In this article, we only consider contention-free DRP to support QoS traffic.

In [14], two analytical models for resource assignment in WiMedia MAC are proposed: subframe-fit and isozone-fit reservations. The subframe-fit scheme only uses request sizes and delay requirements, whereas the isozone-fit scheme does block sizes and locations recommended in [15]. They also suggest improvements to the isozone-fit algorithm by introducing cross-isozone allocation and on-demand compaction.

Adaptive multiuser (MU) spectrum allocation methods have been investigated in $[16,17]$. They allow users to share available resources by exploiting the effective signal-to-interference plus noise ratio and priority level, depending on throughput, delay, and packet error rate. They apply cross-layer approaches for the PHY and MAC layer designs that use the channel state information and service differentiation.

The WiMedia standard adopts MB-OFDM where signal transmission uses only one of the three bands at a symbol time. This means that the standard scheme does not exploit the bandwidth fully. In this article, we

\section{Springer}


increase the wireless bandwidth three times using the three bands together, which is enabled by synchronizing devices in a piconet. This provides the benefit of increasing the number of multimedia flows to be serviced at a time. To deal with the enlarged bandwidth in supporting various QoS traffic types, we consider appropriate resource allocation policies too.

The remainder of the article is organized as follows. In Section 2, we briefly overview WiMedia PHY and MAC, and propose the synchronized MB-OFDM in Section 3. We consider the resource allocation algorithms to deal with the enlarged bandwidth in Section 4. Then, we apply the proposed allocation policies with some operation rules for prioritized QoS traffic support in Section 5, and present simulation results in Section 6, followed by concluding remarks in Section 7 .

\section{Background}

We overview the WiMedia specification with regard to PHY and MAC layers, MB-OFDM, and time-frequency code (TFC).

\subsection{WiMedia PHY and MAC}

ECMA specified WiMedia PHY and MAC, called ISO (International Organization for Standardization)-based ECMA-368 [2]. The standard uses the spectrum between 3.1 and $10.6 \mathrm{GHz}$ and supports the data rates of 53.3, 80, 106.7, 160, 200, 320, 400, and 480 Mbps. The spectrum is divided into 14 bands with each bandwidth of $528 \mathrm{MHz}$. The consecutive three bands form one band group except the last two bands that form the last fifth group. And frequency-domain and timedomain spreading, forward error correction with convolutional codes are used.

WiMedia MAC uses a superframe that contains 256 medium access slots (MASs), and coordinates frame transmission in a distributed manner. The superframe structure consists of two periods: beacon period (BP) and data transfer period (DTP) as shown in Figure 1. The BP starts with the beacon period start time (BPST)

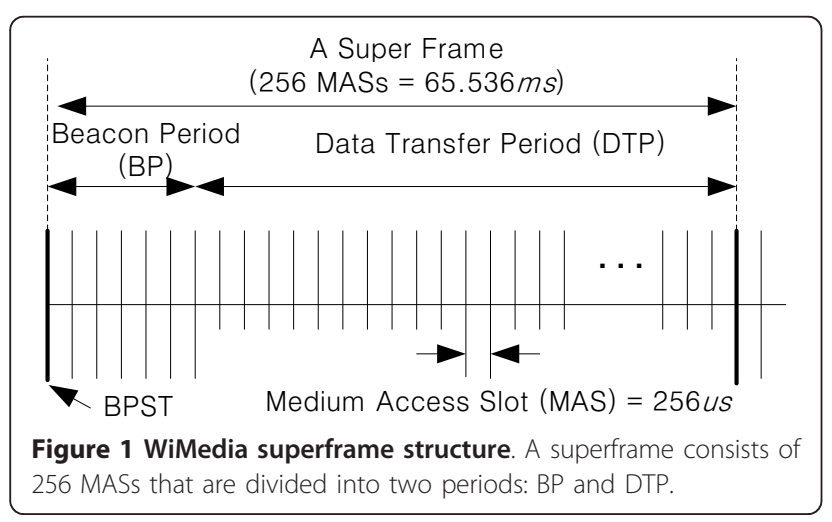

which is the start time of the first MAS in the BP, followed by the superframe. All the devices resynchronize their interval timers obtained from received beacons with each other at the beginning of every superframe. Then, each device sends a beacon frame at its designated time slot and listens to all the beacon frames from other devices. In the DTP, MASs are accessible by PCA or DRP. PCA uses carrier sense multiple access with collision avoidance and priority for the channel access of asynchronous services. Whereas, DRP uses reservation-based TDMA for isochronous i.e. strict QoS services.

\subsection{MB-OFDM}

MB-OFDM is a combination of frequency hopping and OFDM. The frequency hopping allows only one of the three bands to be used at each symbol time as shown in Figure 2. ${ }^{\text {a }}$ There are a total of 128 subcarriers in each band. The numbers of data, pilot, null, and guard subcarriers are $100,12,6$, and 10 , respectively. The frequency hopping provides the frequency diversity and mitigates the co-channel interference between neighboring piconets which operate independently, and exploits the maximum transmit power per device following the regulation of Federal Communications Commission (FCC).

\subsection{TFC}

The coded information is spread with TFCs that are classified into three types: time-frequency interleaving (TFI), TFI2, and fixed frequency interleaving (FFI) as shown in Table 1. The coded data are interleaved over one, two, and three band(s) in FFI, TFI2, and TFI, respectively. The TFCs are designed to allow the average collision probability of $1 / 3$ at maximum between two TFCs, since they are not always orthogonal. Table 2 shows the collision probabilities between two TFCs in band group 1.

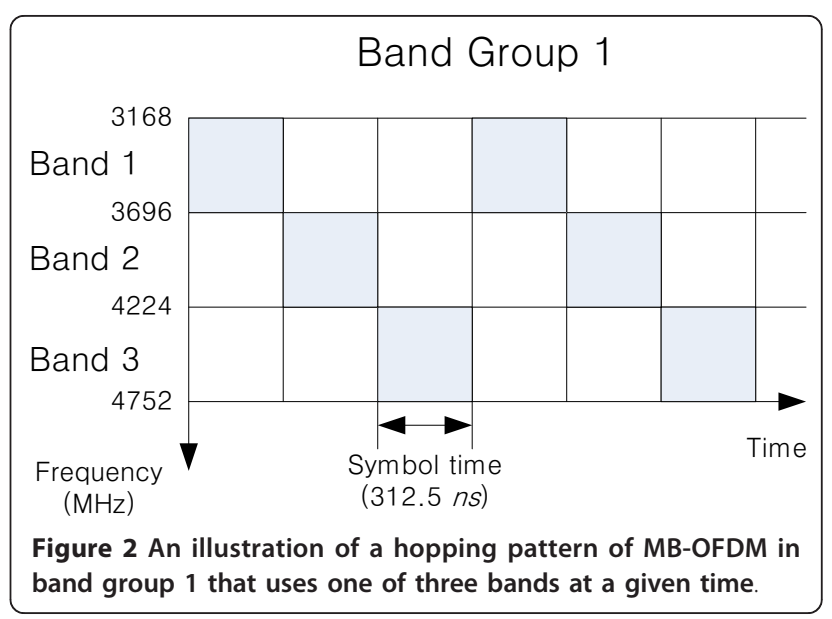


Table 1 Time-frequency codes for band group 1 in ECMA368 [2]

\begin{tabular}{cccccccc}
\hline TFC number & Types & \multicolumn{7}{c}{ Band ID for TFC } \\
\hline 1 & $\mathrm{TFI}$ & 1 & 2 & 3 & 1 & 2 & 3 \\
2 & $\mathrm{TFI}$ & 1 & 3 & 2 & 1 & 3 & 2 \\
3 & $\mathrm{TFI}$ & 1 & 1 & 2 & 2 & 3 & 3 \\
4 & $\mathrm{TFI}$ & 1 & 1 & 3 & 3 & 2 & 2 \\
5 & $\mathrm{FFI}$ & 1 & 1 & 1 & 1 & 1 & 1 \\
6 & $\mathrm{FFI}$ & 2 & 2 & 2 & 2 & 2 & 2 \\
7 & $\mathrm{FFI}$ & 3 & 3 & 3 & 3 & 3 & 3 \\
8 & $\mathrm{TFI} 2$ & 1 & 2 & 1 & 2 & 1 & 2 \\
9 & $\mathrm{TFI} 2$ & 1 & 3 & 1 & 3 & 1 & 3 \\
10 & $\mathrm{TFI} 2$ & 2 & 3 & 2 & 3 & 2 & 3 \\
\hline
\end{tabular}

\section{System model}

We model MU MB-OFDM to exploit three bands at each symbol time. To realize this model in a piconet, we propose to synchronize three concurrent transmissions at each MAS boundary time to overcome the clock drift. Moreover, we consider imperfect synchronization and some issues in applying the model for multi-piconet environments.

\subsection{MU MB-OFDM}

The conventional MB-OFDM uses only one band among the three in a band group at each symbol time. However, the synchronization of devices in a piconet can make it possible to use three bands concurrently, thereby tripling the wireless bandwidth compared to the standard scheme. The synchronization helps to avoid interference from other devices in a piconet. The MU MB-OFDM selects a TFC in TFI or TFI2, and shifts it by some OFDM symbol times to create two or three orthogonal TFCs that can be used together.

Specifically, the numbers of the shift are 0, 1, 2 at TFC1 and TFC2, 0, 2, 4 at TFC3 and TFC4, and 0, 1 at TFC8, TFC9, and TFC10. ${ }^{\mathrm{b}}$ The use of three shifted TFCs brings the gain of the frequency diversity.

Each device in the MU MB-OFDM uses the same transmit power as in the conventional MB-OFDM because each device should conform the regulation of

Table 2 Collision probabilities between TFCs in band group 1

\begin{tabular}{ccccccccc}
\hline TFC \# & $\mathbf{1 - 4}$ & $\mathbf{5}$ & $\mathbf{6}$ & $\mathbf{7}$ & $\mathbf{8}$ & $\mathbf{9}$ & $\mathbf{1 0}$ & Avg. prob. \\
\hline $1-4$ & $1 / 3$ & $1 / 3$ & $1 / 3$ & $1 / 3$ & $1 / 3$ & $1 / 3$ & $1 / 3$ & $1 / 3$ \\
5 & $1 / 3$ & 1 & 0 & 0 & $1 / 2$ & $1 / 2$ & 0 & $1 / 3$ \\
6 & $1 / 3$ & 0 & 1 & 0 & $1 / 2$ & 0 & $1 / 2$ & $1 / 3$ \\
7 & $1 / 3$ & 0 & 0 & 1 & 0 & $1 / 2$ & $1 / 2$ & $1 / 3$ \\
8 & $1 / 3$ & $1 / 2$ & $1 / 2$ & 0 & $1 / 2$ & $1 / 4$ & $1 / 4$ & $1 / 3$ \\
9 & $1 / 3$ & $1 / 2$ & 0 & $1 / 2$ & $1 / 4$ & $1 / 2$ & $1 / 4$ & $1 / 3$ \\
10 & $1 / 3$ & 0 & $1 / 2$ & $1 / 2$ & $1 / 4$ & $1 / 4$ & $1 / 2$ & $1 / 3$ \\
\hline
\end{tabular}

FCC. Figure 3 shows an example of TFC patterns in the MU MB-OFDM with three devices transmitting together at a given time.

\subsection{Synchronization}

In the BP, every node in a piconet is awake and broadcasts its own beacon at its predetermined slot. Each node maintains a table of timing differences between the actual arrival times of each neighbor's beacon by simply synchronizing with the slowest device in the BP. The expected arrival time is calculated based on the BPST.

In the DTP, concurrent transmissions should be synchronized to avoid inter symbol interference between consecutive adjacent transmissions. One OFDM symbol time is $312.5 \mathrm{~ns}$ with the fast Fourier transform time of $242.42 \mathrm{~ns}$ and the zero-padded suffix duration of 70.08 ns, of which function is to overcome the multi-path effect and give time for frequency hopping [2].

We assume that the crystal oscillator has a clock of 4224 MHz. Then, the maximum clock drift is given by

$$
\text { MaxDrift }=2 \times \text { mClockAccuracy } \times \text { SyncInterval, }
$$

where $m$ ClockAccuracy is the clock drift set to 20 PPM (parts per million) and SyncInterval the synchronization time of a device in the DTP. MaxDrift is about $2.62 \mu \mathrm{s}$ for each transmission pair in a superframe if all the nodes are synchronized in the BP.

There is a guard interval, i.e. mGuardTime $=12 \mu \mathrm{s}$, between two adjacent MAS boundary times to overcome the clock drift in the conventional MAC policy. Consequently, RX nodes are ready to listen to signals prior to mGuardTime at their reserved MAS boundary times.

However, concurrent transmissions scheduled at the same MAS with differently shifted TFCs can arrive prior to the MAS boundary time within mGuardTime simultaneously, when all the devices are synchronized in the

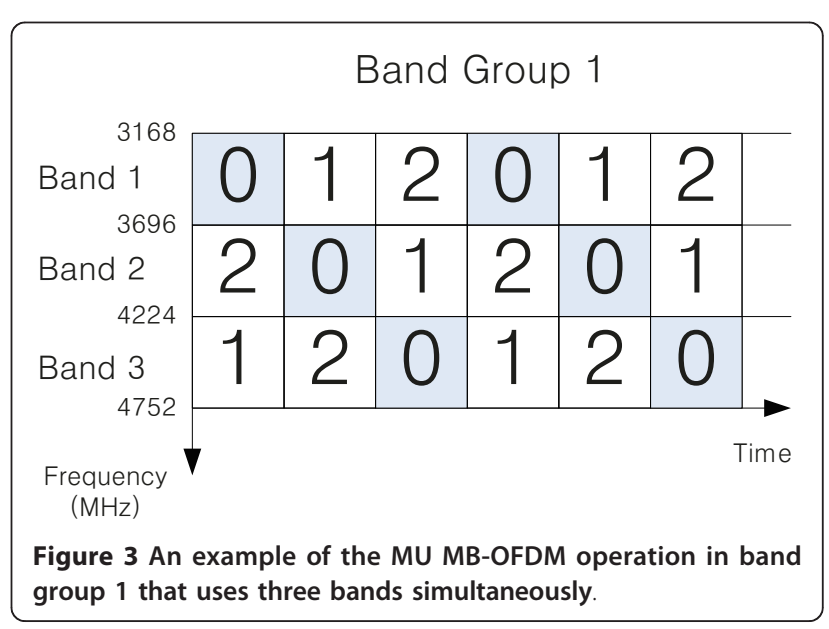


BP. To solve this problem, TX-RX pairs have to listen first to the hopping pattern for the duration of OFDM symbol time, and then transmit their signals according to their scheduled hopping patterns. Each TX-RX pair already knows the hopping patterns of other TX nodes from hearing beacons in the BP.

\subsection{Implementation}

For a practical implementation, we propose to use an $M U$ synchronization at the MAS boundary as shown in Figure 4.

The frame structure has the PLCP protocol data unit (PPDU) that consists of physical layer convergence protocol (PLCP) preamble, PLCP header, and PHY service data unit. The PLCP preamble has two distinct parts: a unique synchronization sequence and a channel estimation sequence. It helps the receiver in timing synchronization, carrier-offset recovery, and channel estimation. In our proposed MU-synchronization, TX0 with 0 shift starts to transmit a PPDU first based on its local timer and the other nodes, i.e. TX1, TX2, RX0, RX1, and RX2, start to listen to the synchronization sequence of TX0 for the synchronization with their local timers. The transmitters, TX1 and TX2, start to transmit their PPDUs with their shifted TFCs after the synchronization. Then, the TX-RX pairs can communicate synchronously.

\subsection{Imperfect synchronization}

There is still a timing offset because of the unavoidable propagation delay between two nodes. The maximum timing offset between two transmitters at a receiver is shown in Figure 5 and expressed as

$$
d_{\text {prop } \max }=\frac{2 D_{\max }}{c},
$$

where $D_{\max }$ is the maximum distance between two nodes in a piconet, and $c$ is the speed of light. The timing offset between a TX and the other TX, measured at an RX, is $d_{\text {prop }} \in\left[0, d_{\text {prop,max }}\right]$.

WiMedia UWB can support the ranging capability that calculates the distance between two nodes with an accuracy of $\pm 60 \mathrm{~cm}$ or better. The ranging is performed by calculating the round trip delay using the two-way time transfer technique. We assume that each device maintains a table for distances to other nodes using this ranging. Then, all the TX nodes can remove $d_{\mathrm{TX}-\mathrm{TX}}$ in Figure 5 by adjusting their local timers using this table. Therefore, the maximum timing offset with ranging is given by

$$
d_{\mathrm{rng}, \max }=\frac{D_{\max }}{c} .
$$

\subsection{Effects of imperfect synchronization}

Though the timing offset can be mitigated by the ranging capability of WiMedia UWB, it cannot be removed perfectly. We consider using the zero-padded prefix in an OFDM symbol time to absorb such timing offset. The zero-padded suffix duration of 70.08 ns in a OFDM symbol serves to mitigate the effects of multi-path and give a

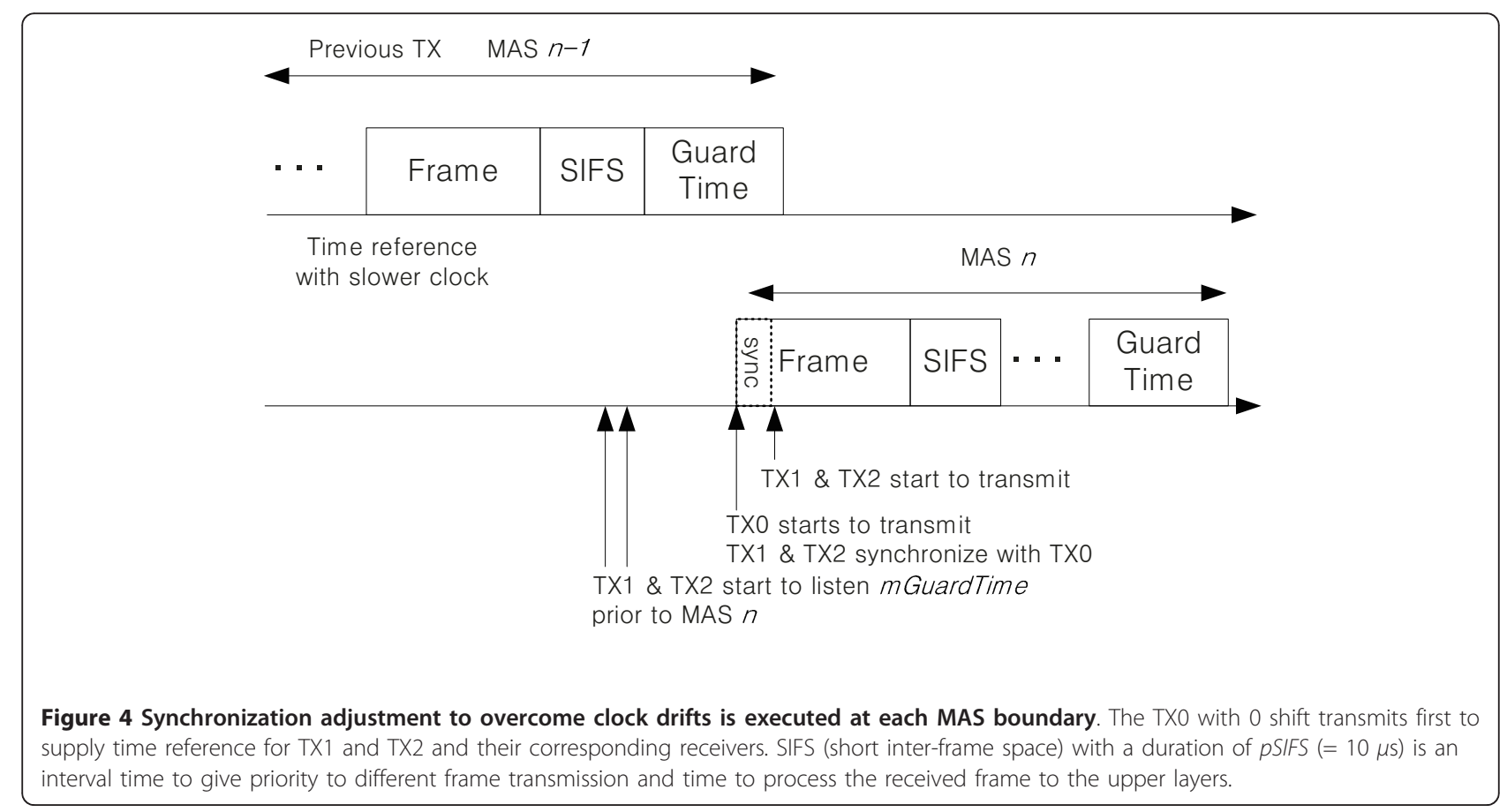




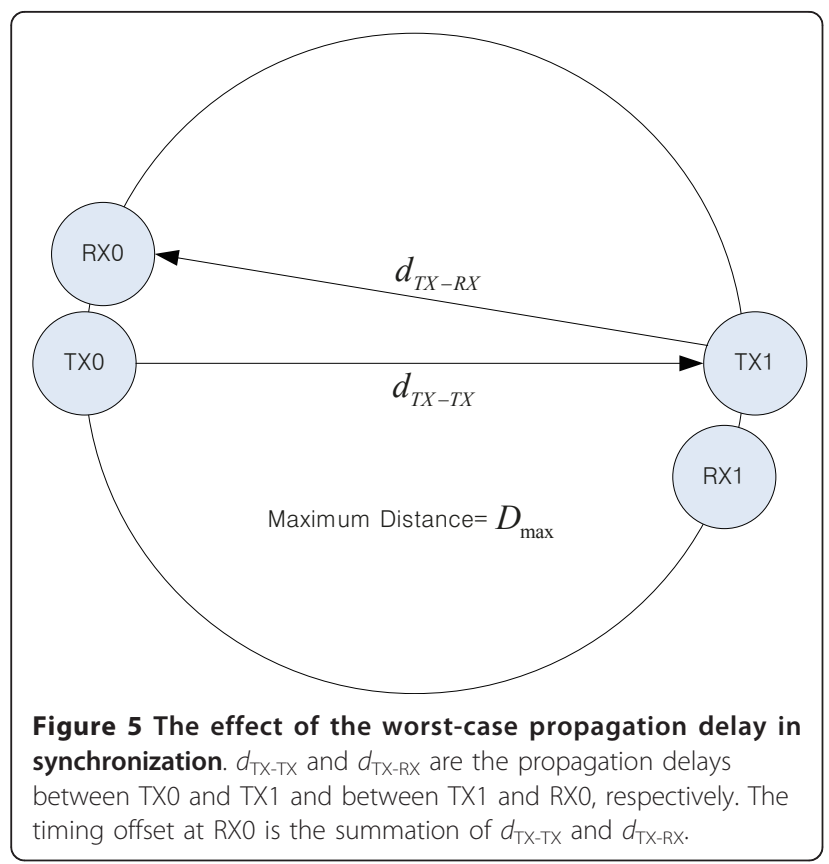

guard time for the band switch, $p$ BandSwitchTime $(=9.47$ $\mathrm{ns})$. And the indoor communication range for multimedia traffics is generally within a few meters, resulting in $d_{\text {rng, }}$ max to be below a few ns delay, e.g. $10 \mathrm{~ns}$ at $3 \mathrm{~m}$.

However, the received signal will be degraded if the effects of multi-path and the propagation delay are not mitigated sufficiently by the zero-padded suffix duration. In this case, the TX-RX pair lowers their transmission rate based on packet error rate in practice to overcome the effects of imperfect synchronization, requiring more wireless resources. Therefore, this imperfect synchronization degrades the network throughput.

\subsection{Multi-piconet environments}

In normal operation, there is no interference in a piconet if all the nodes are synchronized and scheduled in the MU MB-OFDM. But the interference is not avoidable if the network is heavily loaded in a multi-piconet environment. It happens when some bands are occupied again by neighboring piconets at a given time.

In the conventional MB-OFDM, several methods such as transmit power control, band group change, TFC change, and exclusive time reservation have been proposed to mitigate the interferences from neighboring networks [18-21]. In this context, we use the band group change to avoid the interferences from other piconets. Our scheme can support 14 concurrent users at a time in the UWB spectrum, i.e. a user per band, without creating interference. ${ }^{\mathrm{C}}$

Different from ours, the standard scheme can support five users at a time, i.e. a user per band group. This means our scheme can accommodate about three times more users than the standard scheme. To apply our scheme to a multi-piconet environment, we need to adopt a solution to a distributed vertex coloring problem with five colors, i.e. a different color for each band group. Several solutions to this problem have been proposed and analyzed [22-24]. The detailed discussion about the coloring problem is beyond the scope of this article.

\section{Resource allocation}

In this section, we review the conventional 2-dimensional (2D) resource allocation scheme to assign 256 MASs in MB-OFDM, and consider 3-dimensional (3D) allocation schemes to deal with the increased $3 \times 256$ MASs in MU MB-OFDM.

\subsection{Conventional 2D resource allocation}

The 2D structure of $16 \times 16$ MASs in a superframe has been proposed for MAS allocation [15]. The contiguous 16 MASs are grouped into an allocation zone, called zone. There are 16 zones in column. We denote the zones by $\mathcal{Z}_{0}$. to $\mathcal{Z}_{15}$. $\mathcal{Z}_{0}$ is reserved for $\mathrm{BP}$, and the other 15 zones are grouped into four subsets, called isozones. We denote the set of zones with isozone $j$ by $\mathcal{I}_{j}$ that has $2^{j} \quad$ zones. That is, $\mathcal{I}_{0}=\left\{\mathcal{Z}_{8}\right\}$, $\mathcal{I}_{2}=\left\{\mathcal{Z}_{2}, \mathcal{Z}_{6}, \mathcal{Z}_{10}, \mathcal{Z}_{14}\right\} \mathcal{I}_{2}=\left\{\mathcal{Z}_{2}, \mathcal{Z}_{6}, \mathcal{Z}_{10}, \mathcal{Z}_{14}\right\}, \quad$ and $\mathcal{I}_{3}=\left\{\mathcal{Z}_{1}, \mathcal{Z}_{3}, \mathcal{Z}_{5}, \mathcal{Z}_{7}, \mathcal{Z}_{9}, \mathcal{Z}_{11}, \mathcal{Z}_{13}, \mathcal{Z}_{15}\right\}$

Since an MAS has the duration of $256 \mu \mathrm{s}$, each zone is separated by $4.096 \mathrm{~ms}$ from each neighboring one. Higher-indexed isozones are used to support services with smaller service intervals, i.e. tight QoS requirements. For instance, the service intervals of $\mathcal{I}_{0}$ and $\mathcal{I}_{3}$ are $16 \times 4.096$ and $2 \times 4.096 \mathrm{~ms}$, respectively. When a flow with QoS requirements enters the network, it indicates its service requirements by an isozone number and the number of required MASs in a superframe.

The number of available MASs with isozone $j$, denoted by $m_{j}$, is expressed as

$$
m_{j}=2^{j} \gamma_{j}, \quad \gamma_{j} \in\{0, \ldots, 16\},
$$

where $y_{j}$ is the number of available MASs in each zone with $\mathcal{I}_{j}$. The MAS allocation follows the symmetric assignment property [15]. We shown an example of 2D MAC resource allocation in Figure 6.

\subsubsection{D MAC policy}

This policy tries to find available resources in a higherindexed isozone, which meets the requested maximum delay bound, when there is an insufficient number of MASs in the requested isozone. The 2D MAC policy is expressed as follows:

$$
\begin{aligned}
P_{2 \mathrm{D}}\left(r_{i}\right)= & \min \left\{2^{j *} x \mid r_{i} \leq 2^{j *} x \leq m_{j *}\right\}, \\
\text { s.t. }{ }^{*}= & \min \left\{j \mid r_{i} \leq m_{j}, i \leq j \leq 3\right\}, \\
& x \in\{0, \ldots, 16\},
\end{aligned}
$$




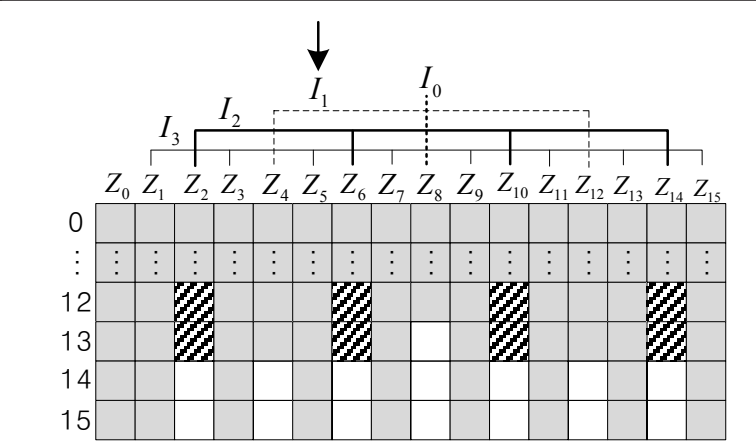

Figure 6 The 2D structure of MASs in the conventional MBOFDM. The number of assigned MASs when $r_{1}=6$ is 8 with $\mathcal{I}_{2}\left(m_{2}=16\right)$. In this example, the available MASs in $\mathcal{I}_{1}$ are insufficient $\left(m_{1}=4\right)$. Hence, the assignment for $\mathcal{I}_{2}$ is needed, and two MASs are excessively allocated.

where $P_{2 \mathrm{D}}$ is the number of assigned MASs in the 2D MAC policy, $r_{i}$ the number of requested MASs in $\mathcal{I}_{i}$ specified by a QoS flow, and $x$ the number of selected MASs in each zone with $\mathcal{I}_{j *}$. Note that the assigned MASs can be more than the requested MASs because of the symmetric assignment property. The MASs to be allocated are evenly distributed over the zones with the same requested isozone for the convenience of future reservation.

\subsection{D resource allocation}

Against the standard 2D allocation of $16 \times 16$ MASs, our proposed allocation schemes handle the 3D structure of $3 \times 16 \times 16$ MASs. This structure comes from the MU MB-OFDM that uses the three bands. We denote the three superframes with 0,1 , and $2 \operatorname{shift}(\mathrm{s})$ of OFDM symbol time by $S F_{0}, S F_{1}$, and $S F_{2}$, respectively. ${ }^{\text {d }}$ This implies that the standard MB-OFDM uses $S F_{0}$ only.

In the 2D MAC policy, if there are not enough MASs in the requested isozone of a superframe, each TX node searches for MASs from other higher-indexed isozones. In our MU MB-OFDM scheme, as we have the 3D resource structure, we can consider three types of resource assignment policies: SF (SuperFrame)-first pol$i c y$ tries available resources sequentially from equal and next higher-indexed isozones in $S F_{0}$ first, IZ (IsoZone)first policy tries the requested isozone first over the three SFs, and SIZ (Shared-IZ) policy tries resources from all the isozones and $S F$ s exhaustively. When a resource request is given, SIZ policy can partially assign MASs from an isozone and then additional MASs from other isozones over the three $S F$ s. We explain these three policies in detail.

\subsubsection{SF-first policy}

To find available MASs, this policy tries equal and then higher-indexed isozones in $S F_{0}$ first. If not found, it tries $S F_{1}$ and $S F_{2}$ sequentially until it finds the requested resources, as shown in Figure 7. This policy is expressed as

$$
\begin{aligned}
P_{S F}\left(r_{i}\right)= & \min \left\{2^{j^{*}} x \mid r_{i} \leq 2^{j^{*}} x \leq m_{j *, l *}\right\}, \\
\text { s.t.j }{ }^{*}= & \min \left\{j \mid\left(j, l^{*}\right) \in \mathcal{A}_{S F}\right\}, \\
l^{*}= & \min \left\{l \mid(j, l) \in \mathcal{A}_{S F}\right\}, \\
\mathcal{A}_{S F}= & \left\{(j, l) \mid r_{i} \leq m_{j, l}, l \in\{0,1,2\}, i \leq j \leq 3\right\}, \\
& x \in\{0, \ldots, 16\},
\end{aligned}
$$

where $P_{S F}$ is the number of assigned resources, $\mathcal{A}_{S F}$ the set of available isozones $j$ with each $S F$, and $m_{j, l}$ the available MASs with $\mathcal{I}_{j}$ and $S F_{l}$.

\subsubsection{IZ-first policy}

This policy assigns resources to the requested isozone first and searches through the three SFs. If there exist insufficient MASs at the requested isozone over the three $S F$ s, this policy tries next higher-indexed isozones until found. The searching sequences are depicted in Figure 8 and expressed as

$$
\begin{aligned}
P_{I Z}\left(r_{i}\right)= & \min \left\{2^{3^{*}} x \mid r_{i} \leq 2^{j^{*}} x \leq m_{j^{*}, l^{*}}\right\}, \\
\text { s.t. } l^{*}= & \min \left\{l \mid\left(j^{*}, l\right) \in \mathcal{A}_{I Z}\right\}, \\
j^{*}= & \min \left\{j \mid(j, l) \in \mathcal{A}_{I Z}\right\}, \\
\mathcal{A}_{I Z}= & \left\{(j, l) \mid r_{i} \leq m_{j, l}, l \in\{0,1,2\}, i \leq j \leq 3\right\}, \\
& x \in\{0, \ldots, 16\},
\end{aligned}
$$

where $P_{I Z}$ is the number of assigned resources and $\mathcal{A}_{I Z}$ the set of available isozones with each $S F$.

\subsubsection{SIZ policy}

This policy tries cross isozones for MAS allocation if the requested MASs cannot be allocated to one isozone of an $S F$. This is a simply extended version of the 2D cross-IZ allocation scheme for 3D allocation [14]. Given the resource request $r_{i}$, it will be allocated to isozone $j(\geq$ $i)$ that uses the minimum sum of MASs, while meeting the QoS requirements.

When this policy is applied to the case of $r_{1}=6$ in Figure 6 it selects two isozones that have two MASs in

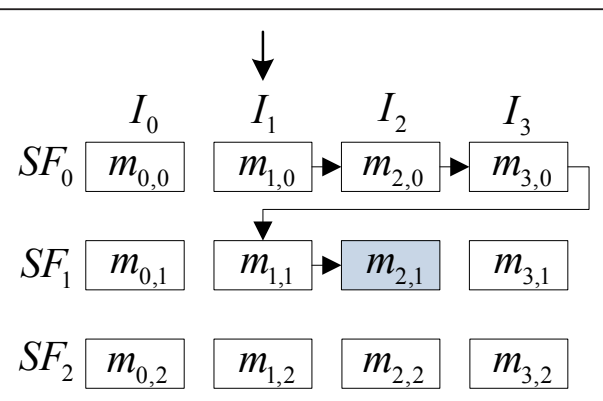

Figure 7 SF-first policy. A flow requires $\mathcal{I}_{1}$ in this case. This policy tries $\mathcal{I}_{1}$ to $\mathcal{I}_{3}$ in $S F_{0}$ to find available resources, and then in $S F_{1}$ and $S F_{2}$, sequentially. In this example, $\mathcal{I}_{2}$ in $S F_{1}$ has available MASs that meet the requirement. 


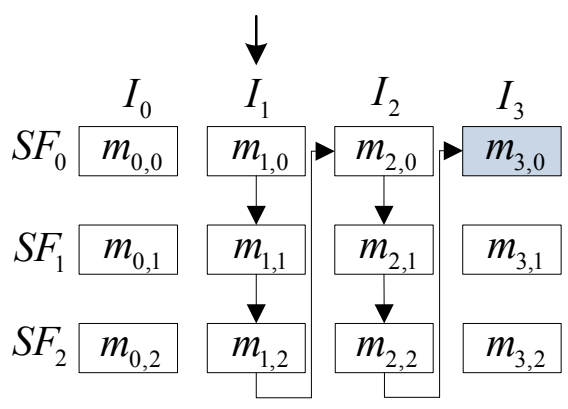

Figure 8 IZ-first policy. A flow requires $\mathcal{I}_{1}$ in this example. This policy tries the same isozone first over the three SFs to assign resources, and finally finds enough resources at $\mathcal{I}_{3}$ in $S F_{0}$.

$\mathcal{I}_{2}$ and four MASs in $\mathcal{I}_{2}$, respectively. The isozones are not necessarily from the same SF. SIZ policy is illustrated in Figure 9 and expressed as

$$
\begin{aligned}
P_{S I Z}\left(r_{i}\right) & =\sum_{j=0}^{3} 2^{j} x_{j}^{*}, \\
\text { s.t. }\left(x_{0}^{*}, x_{1}^{*}, x_{2}^{*}, x_{3}^{*}\right) & =\underset{\left(x_{0}, x_{1}, x_{2}, x_{3}\right) \in A_{\text {SII }}}{\arg \sum_{j=0}^{3} 2^{j} x_{j,},} \\
M_{j} & = \begin{cases}\max \left(m_{j, 0}, m_{j, 1}, m_{j, 2}\right), \quad i \leq j \leq 3, \\
0 & \text { otherwise, }\end{cases} \\
\mathcal{A}_{S I Z}= & \left\{\begin{array}{l}
\left.\left(x_{0}, x_{1}, x_{2}, x_{3}\right) \mid r_{i} \leq \sum_{j=0}^{3} 2^{j} x_{j} \leq \sum_{j=0}^{3} M_{j}\right\}, \\
x_{j} \in\left\{0, \ldots, M_{j} / 2^{j}\right\}, 0 \leq j \leq 3,
\end{array}\right.
\end{aligned}
$$

where $P_{S I Z}$ is the number of assigned resources, $\mathcal{A}_{S I Z}$ the set of feasible combinations of $x_{j}, M_{j}$ the maximum of available MASs in $\mathcal{I}_{j}$ over the three $S F \mathrm{~s}$, and $x_{j}$ the number of selected MASs with $\mathcal{I}_{j}$ in the selected $S F$.

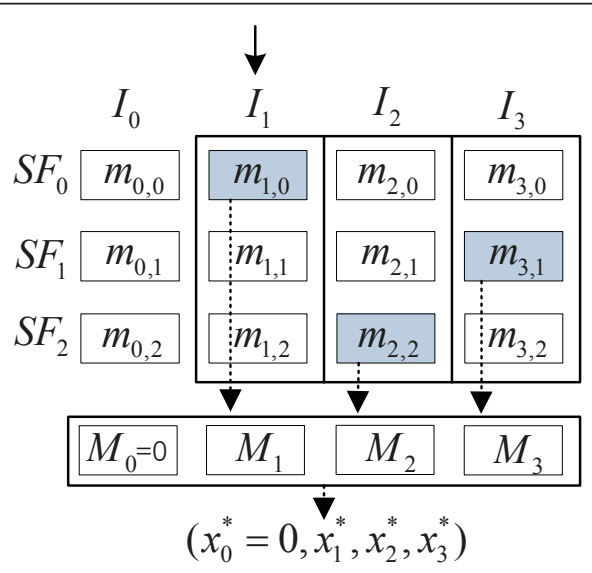

Figure 9 SIZ policy. A flow requires $\mathcal{I}_{1}$ in this case. This policy selects MASs from multiple isozones to accommodate the required MASs. Only one $\mathcal{I}_{j}$ with the most available MASs from each SF is mapped into $M_{j}$. The policy selects a best combination of $x_{j}^{*}$ that minimizes the number of assigned MASs for $r_{i}$.
The search space in this policy is larger than those in SF-first and IZ-first policies, leading to a best combination. For simplicity, we omitted the $S F$ index for $M_{j}$ in (8). To find $x_{j}^{*}$ and its $S F$ index exhaustively, we present an algorithm in Figure 10 as an example.

\section{Resource allocation for prioritized QoS traffic}

In this article, we consider video with low quality (VL), video with high quality $(\mathrm{VH})$, and best effort $(\mathrm{BE})$, and assume that $\mathrm{VL}$ has priority over $\mathrm{VH}{ }^{\mathrm{e}}{ }^{\mathrm{e}} \mathrm{BE}$ has no priority and requirements, and simply tries to take all the available MASs that are unassigned to VL and VH.

\subsection{Priority support}

The resource allocation policy for QoS flows can be preemptive or non-preemptive: a policy is preemptive if a QoS flow can be interrupted by another QoS flow, and non-preemptive otherwise.

\subsubsection{Preemptive policy}

Each QoS flow of VL or VH is assigned to at least one $S F$ with available isozones. We simply dedicate one $S F$ to $\mathrm{VL}$ and two $S F \mathrm{~s}$ to $\mathrm{VH}$, and use the following rules for preemptive QoS operation with ownership.

- VL owns $S F_{0}$, VH owns $S F_{1}$ and $S F_{2}$, but BE has no dedicated $S F$.

- VL and VH occupy any available $S F$ and preempt BE.

- VL can preempt VH in $S F_{0}$, but VH cannot preempt VL in $S F_{1}$ and $S F_{2}$.

- An existing owner of each $S F$ cannot be preempted by other traffic types.

We also consider preemptive QoS operation without ownership.

- VL and VH occupy any available $S F$ without dedicated $S F$ and preempt BE.

- VL can preempt VH over three SFs.

\subsubsection{Non-preemptive policy}

All the QoS flows of VL and VH can use three SFs without being preempted by next incoming QoS flows. However, BE flows still can be preempted by QoS flows.

\subsection{BE service support}

All the unassigned MASs can be allocated for BE services. Incoming $\mathrm{BE}$ flows share available MASs with other existing BE flows in a fair manner, and do not follow the symmetric assignment property.

We propose a Cross-SF allocation policy for BE traffic with an example in Figure 11. We denote the number of available MASs for BE traffic in $S F_{0}, S F_{1}$, and $S F_{2}$ on 


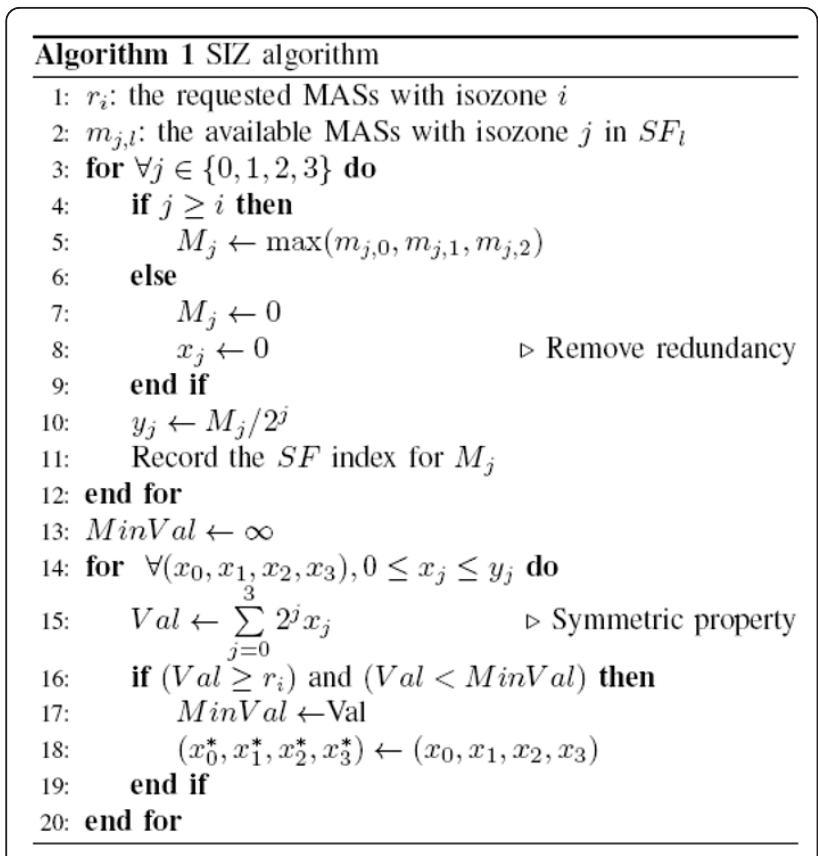

Figure $10 \mathrm{SIZ}$ algorithm.

each MAS index $q \in\{1, \ldots ., M\}$ by $N_{q} \in\{0,1,2,3\}$ and classify the MASs on an SF into as a set of $S_{N_{q}} \subset\{1, \ldots, M\}$, where $M$ is the maximum number of MASs in an $S F$. In Figure 11a, there are no remaining MASs for BE flows at MAS 5 and 7, i.e. $\mathcal{S}_{0}=\{5,7\}$. And other sets are $\mathcal{S}_{1}=\{1,3\}, \mathcal{S}_{2}=\{2,4,8\}$, and $\mathcal{S}_{3}=\{6\}$.

Let us consider $N$ BE flows. As a $\mathrm{BE}$ flow can transmit through only one MAS at a time, the number of assigned MASs to each BE flow $n$ is given by

$$
P_{C S F}(N, n)=\left\{\begin{array}{l}
\sum_{i=1}^{3}\left|S_{i}\right|, \quad N=1, \\
\sum_{i=2}^{3}\left|S_{i}\right|+\left\lfloor\left|S_{1}\right| / 2\right\rfloor+b_{n}, \quad N=2, \\
\left\lfloor\left(\sum_{i=1}^{3} i \times\left|S_{i}\right|\right) / N\right\rfloor+b_{n}, \quad N \geq 3,
\end{array}\right.
$$

where $P_{C S F}(N, n)$ is the number of MASs to be assigned over the three $S F \mathrm{~s}$ for BE flow $n,\left|\mathcal{S}_{i}\right|$ is the number of elements in the set $\mathcal{S}_{i},\lfloor x\rfloor$ is a floor function which maps $x$ to the largest integer not greater than $x$. And $b_{n}$ is a binary variable having 0 or 1 when the input $x$ of $\lfloor x\rfloor$ is not an integer, and 0 otherwise. One MAS will be assigned to BE flow $n$ starting with 1, i.e. $b_{n}=1$, till the remaining MASs are empty if the input $x$ is not an integer.

After calculating $P_{C S F}(N, n)$, each BE flow $n$ occupies resources in a descending order of $N_{q}$ in $S_{N_{q}}$, i.e. $\mathcal{S}_{3}, \mathcal{S}_{2}$, and $\mathcal{S}_{1}$. When two or three MASs are available at a given time, a low-indexed $S F$ is selected.

The first arriving flow 1 in Figure 11a transmits through six MASs sequentially, i.e. $S F_{2}, S F_{0}, S F_{0}, S F_{0}, X$, $S F_{0}, X, S F_{0}$, where $X$ indicates 'not available' MAS at the given time. The number of assigned MAS for flow 1 is 6.

The second arriving flow 2 in Figure 11b has the same number of assigned MASs with flow 1 according to (9): $P_{C S F}(2,1)=5$ and $P_{C S F}(2,2)=5$. At MAS 6 the resource on $S F_{2}$ cannot be assigned to any flow because a BE flow can transmit through only one MAS at a time.

Finally, flow 3 in Figure 11c requests resources and then we get $P_{C S F}(3,1)=4, P_{C S F}(3,2)=4$ and $P_{C S F}(3$, $3)=3$ from (9). Therefore, the Cross-SF allocation policy guarantees the fairness of each $\mathrm{BE}$ flow.

\section{Simulation results}

In simulations, QoS flows are generated with uniformly distributed delay requirements in [10] ms. Each QoS flow comes with a requested isozone corresponding to the delay requirement. VL and VH flows have a uniformly distributed MAS requirement in $[2,10]$ and in [10], respectively. The maximum number of MASs in an $S F$, i.e. $M$, is set to 240 . We ran the simulations 1,000 times with MATLAB [25] and averaged out the results.

\subsection{Case of VL traffic only}

If we only consider the requested MASs without the symmetric assignment, about 40 flows are supportable at maximum in the standard MB-OFDM. We compare the throughput of the proposed MU MB-OFDM with that of the standard MB-OFDM. Then, we measure the ratio of redundant MASs and the number of blocked flows for each assignment policy.

\subsubsection{Throughput}

Figure 12 shows that the throughput in the MU MBOFDM is saturated at three times as high load as that in the standard MB-OFDM. At the load of 26 flows, the throughput of the standard MB-OFDM is saturated. The throughputs using SF-first, IZ-first, and SIZ policies in the MU MB-OFDM are saturated at 80,80 , and 110 flows, respectively. These policies have not reached the maximum capacity yet because of the property of symmetric MAS assignment.

\subsubsection{Redundant MASs}

Owing to the symmetric assignment property, some allocated MASs are actually unused and wasted. Figure 13 shows that the standard 2D policy has the highest ratio of redundant MASs. This is because it has only one superframe, thereby having a small number of possible MAS allocation combinations for the requested isozone.

In the SF-first policy, the ratio of redundant MASs starts to decrease at the loads of 26 and 54 flows where the policy starts to allocate resources with each additional SF. The IZ-first policy shows lower ratio than the SF-first policy as the IZ-first policy assigns MASs to the requested isozone as much as possible. The SIZ policy 


\begin{tabular}{|c|c|c|c|c|c|c|c|c|}
\hline MAS $\rightarrow$ & $\Rightarrow \quad 1$ & 2 & 3 & 4 & 5 & 6 & 7 & 8 \\
\hline$S F_{0}$ & 1 & BE1 & BE1 & BE1 & 1 & BE1 & 1 & BE1 \\
\hline$S F_{1}$ & 1 & & 1 & 1 & 1 & & 1 & 1 \\
\hline$S F_{2}$ & BE1 & 1 & 1 & & 1 & & 1 & \\
\hline
\end{tabular}

(a) $\mathrm{N}=1$

\begin{tabular}{|c|c|c|c|c|c|c|c|}
\hline MAS $\rightarrow 1$ & 2 & 3 & 4 & 5 & 6 & 7 & 8 \\
\hline$S F_{0}$ & BE1 & BE2 & BE1 & 1 & BE1 & 1 & BE1 \\
\hline$S F_{1}$ & BE2 & 1 & 1 & 1 & BE2 & 1 & 1 \\
\hline BE1 & 1 & 1 & BE2 & 1 & & 1 & BE2 \\
\hline
\end{tabular}

(b) $\mathrm{N}=2$

\begin{tabular}{|c|c|c|c|c|c|c|c|}
\hline MAS $\rightarrow 1$ & 2 & 3 & 4 & 5 & 6 & 7 & 8 \\
\hline$S F_{0}$ & BE1 & BE3 & BE1 & 1 & BE1 & 1 & BE1 \\
\hline$S F_{1}$ & BE2 & 1 & 1 & 1 & BE2 & 1 & 1 \\
\hline BE3 & 1 & 1 & BE2 & 1 & BE3 & 1 & BE2 \\
\hline
\end{tabular}

(c) $\mathrm{N}=3$

Figure $11 \mathrm{BE}$ resource assignment in the Cross-SF allocation policy $(\boldsymbol{M}=\mathbf{8})$. The set of unused MASs over the three SFs contains candidate MASs for BE flows. The shaded rectangles marked with ' 1 ' are occupied by existing QoS flows. The assigned MASs for all the BE flows are balanced as N grows: flow 1 has 6 MASs in (a), flows 1 and 2 have the same 5 MASs in (b), and flows 1, 2, and 3 have 4, 4, and 3 MASs, respectively in (c).

has the least ratio of redundant MASs. The use of multiple isozones over the three $S F$ s in this policy reduces redundant MAS allocation compared to that of single isozone over the SF-first and IZ-first policies.

The ratio of redundant MASs in the SIZ policy starts to decrease at about 80 flows. We can explain this as follows. First, higher-indexed resources, i.e. having shorter service intervals, become candidates for the resource assignment more frequently. Therefore, higherindexed resources tend to be consumed earlier than lower-indexed resources. This tendency causes higherindexed flows to be blocked more often compared to lower-indexed flows. Second, lower-indexed resources have a lower number of symmetric zones according to (4). This leads this policy to have the lowest ratio of redundant MASs at above 80 flows.

\subsubsection{Blocked flows}

A flow will be blocked if the requested resources are not available. The number of blocked flows in the 3D MAC policies is smaller than that in the conventional 2D policy as shown in Figure 14. The ratio of blocked flows in the proposed 3D policies starts to smoothly increase at above 80 flows, whereas that in the conventional 2D policy rapidly increases at above 26 flows. The SIZ policy shows the lowest ratio of blocked flows.

\subsection{Case of $\mathrm{VL}, \mathrm{VH}$, and $\mathrm{BE}$ traffics}

Flows of $\mathrm{VL}, \mathrm{VH}$, and $\mathrm{BE}$ are generated with the equal probability. We apply the $S I Z$ policy with the preemptive policy for VL and VH flows, and the Cross-SF allocation policy for BE flows. Then, we measure throughput and numbers of serviced, blocked, and dropped flows.

\subsubsection{Throughput}

Figure 15 shows the number of allocated MASs for each traffic type. BE flows occupy most MASs in the beginning owing to their tendency of unlimited resource use. As the numbers of $\mathrm{VL}$ and $\mathrm{VH}$ flows increase, BE flows achieve lower throughput, and VL and VH flows higher 


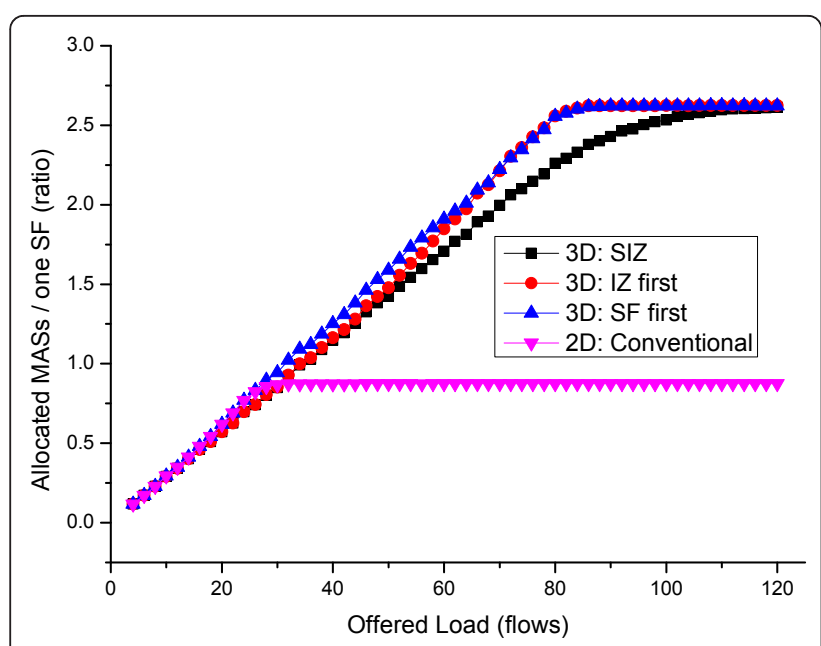

Figure 12 Throughput in the case of VL traffic only. The throughputs of the standard MB-OFDM with the 2D MAC policy and the proposed MU MB-OFDM with SF-first and IZ-first policies are saturated at the loads of 26 and 80 flows, respectively.

throughput. As VL flows have high priority in the preemptive policy with ownership, they are allowed to preempt on-going $\mathrm{VH}$ flows that are being serviced in $S F_{0}$. For this reason, the number of assigned MASs for $\mathrm{VH}$ flows starts to be lowered from the load of 60 flows, and then saturated. However, the number of assigned MASs for VH flows in the preemptive policy without ownership decreases continuously while VL flows achieve higher throughput.

Even when the network is heavily crowded by all the traffic types, BE flows still have a chance to use fragmented MASs that are left over from the allocation for $\mathrm{VL}$ and VH flows.

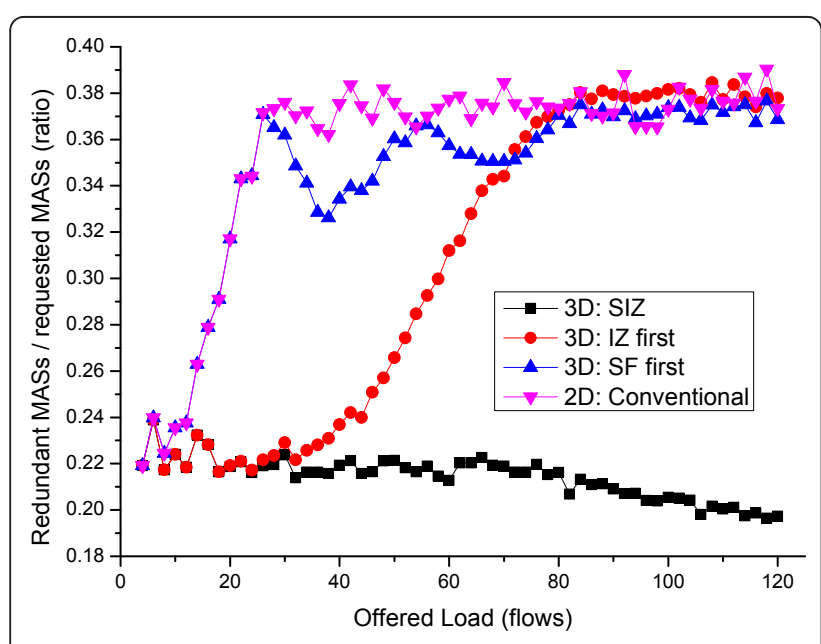

Figure 13 Ratio of redundant MASs in the case of VL traffic only. The SIZ policy has the least ratio of redundant MASs while the 2D MAC policy shows the highest.

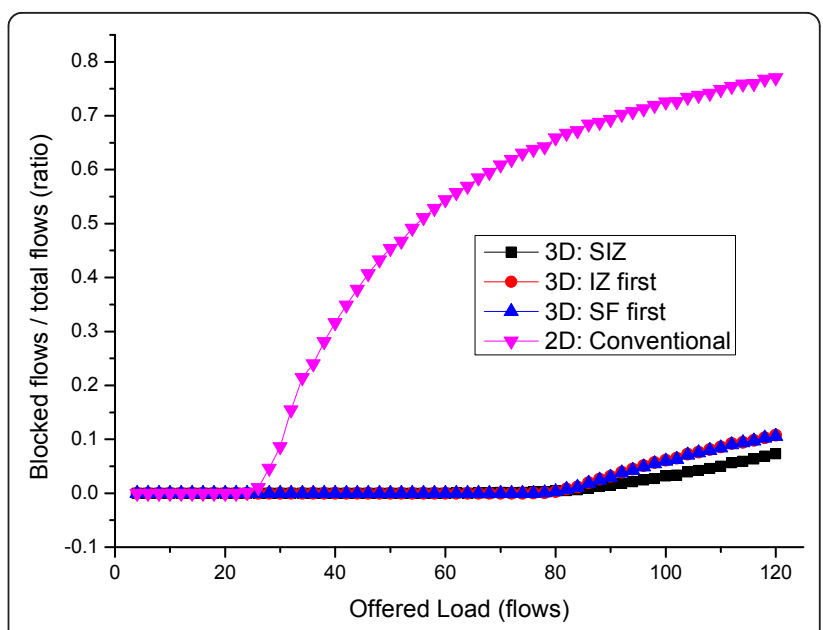

Figure 14 Ratio of blocked flows in the case of VL traffic only. The SIZ policy shows the least ratio of blocked flows.

\subsubsection{Serviced flows}

Figure 16 shows the number of serviced flows for prioritized QoS data. A BE flow is counted as serviced if at least one MAS is assigned, whereas $\mathrm{VL}$ and $\mathrm{VH}$ flows are not allowed for the allocation of fragmented MASs. When VL flows preempt VH flows over three SFs in the preemptive policy without ownership, the number of VL flows that can be admitted is higher than that in the preemptive policy with ownership where VL flows can preempt on only $S F_{0}$. When the number of $\mathrm{VH}$ flows in the preemptive policy without ownership decreases, the number with ownership is saturated as the offered load increases.

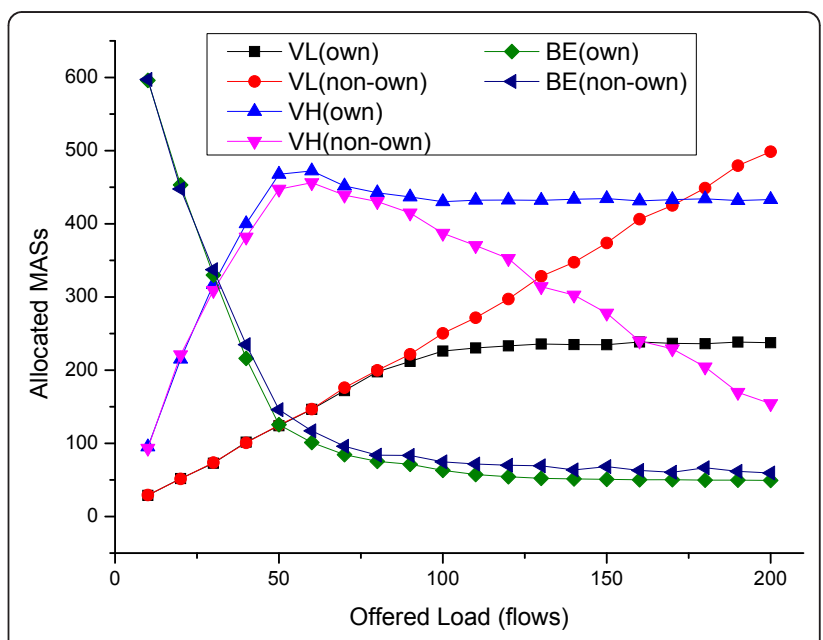

Figure 15 The number of allocated MASs for each traffic type. $\mathrm{VL}, \mathrm{VH}$, and $\mathrm{BE}$ flows are generated with the equal probability. The terms of 'own' and 'non-own' mean that the preemptive policies with and without ownership, respectively. 


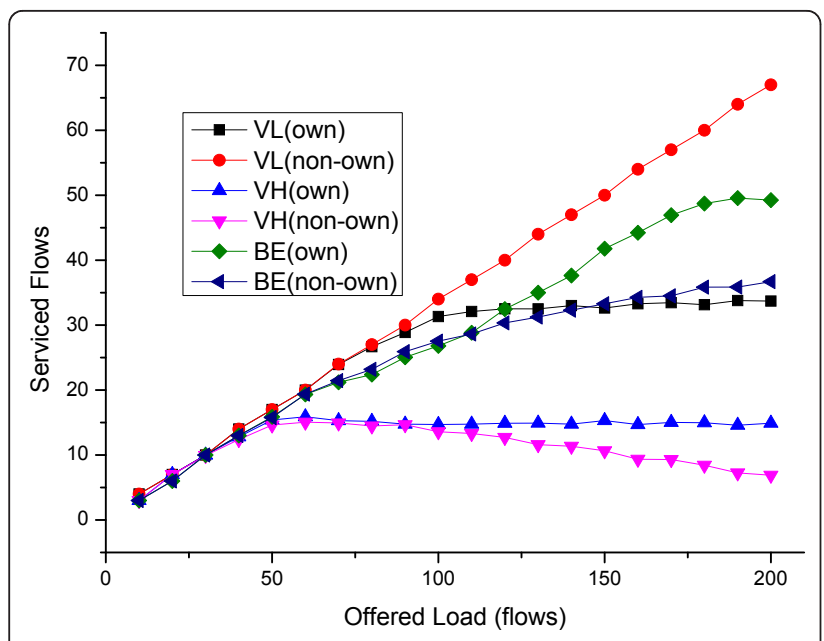

Figure 16 Serviced flows in the case of VL, VH, and BE traffics. $\mathrm{VL}$ and $\mathrm{VH}$ flows in the preemptive policy without ownership show the highest and lowest number of serviced flows, respectively.

\subsubsection{Blocked flows}

As shown in Figure 17 the number of blocked VH flows starts to increase first while that of BE flows last. Again fragmented resources, even one MAS, can be allocated for BE flows. VL flows in the preemptive policy without ownership can still find available resources after preempting $\mathrm{VH}$ flows at the high load.

\subsubsection{Dropped flows}

In the preemptive policy, a flow is dropped if it is preempted by a flow with high priority. VL flows are not dropped because they have the highest priority. The number of dropped $\mathrm{VH}$ and $\mathrm{BE}$ flows starts to rise from the load of 50 flows as shown in Figure 18. The BE and $\mathrm{VH}$ flows in the preemptive policy without ownership are preempted by the $\mathrm{VH}$ flows over three $S F$ s. In this

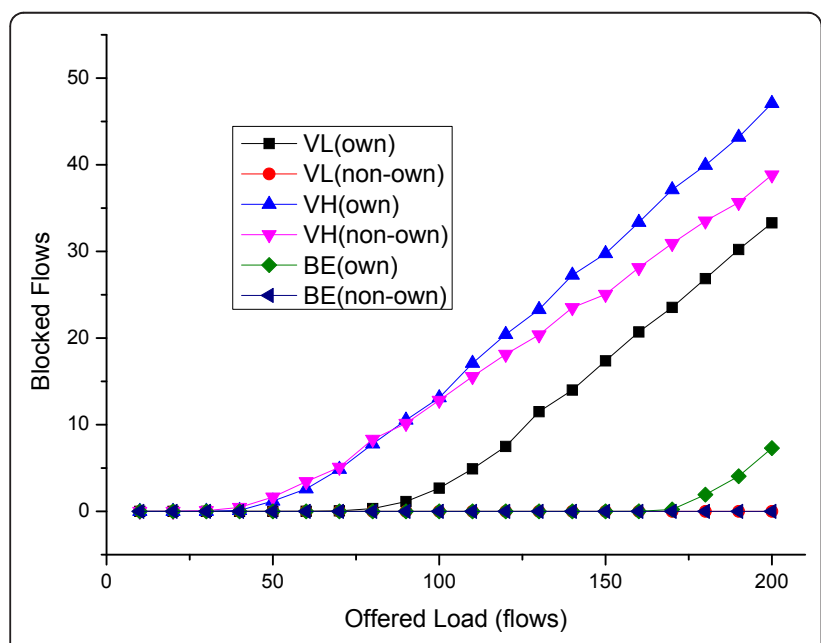

Figure 17 Blocked flows in the case of $\mathrm{VL}, \mathrm{VH}$, and $\mathrm{BE}$ traffics.

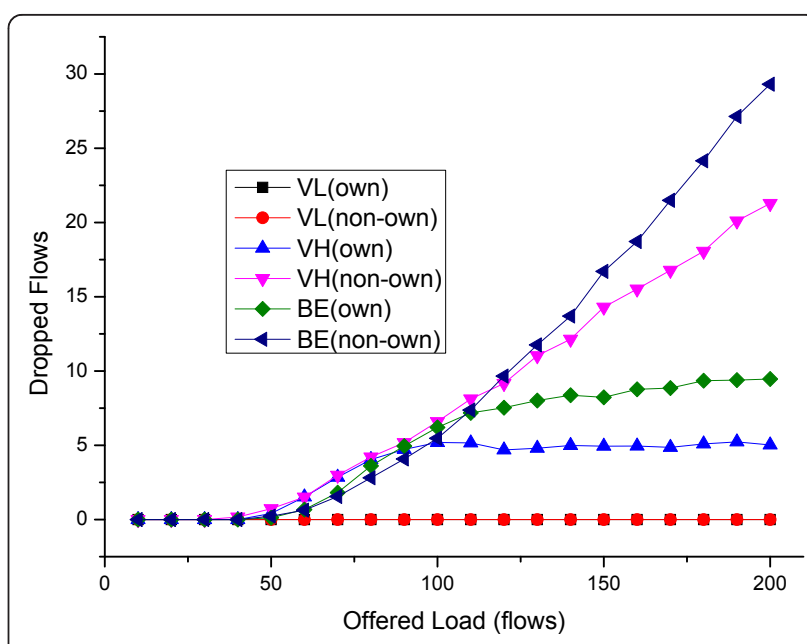

Figure 18 Dropped flows in the case of $\mathrm{VL}, \mathrm{VH}$, and BE traffics. $\mathrm{VL}$ flows will not be dropped because of priority.

regard, the numbers of dropped flows for these flows are higher than those with ownership.

\section{Conclusion}

In this article, we have modeled the MU MB-OFDM using synchronization that provides the merit of three concurrent transmissions for a band group in a piconet. As a result, the proposed MU MB-OFDM triples network throughput compared to the conventional MBOFDM. Then, we considered three 3D resource allocation policies to handle the expanded MAS resources: SF-first, IZ-first, and SIZ policies. The simulations showed that the SIZ policy performs the best in terms of throughput, redundant MASs, and blocked flows.

We also investigated some operation rules with resource allocation policies to support prioritized QoS traffic in the MU MB-OFDM. Extensive simulations presented that the proposed QoS support rules operate well in terms of throughput and numbers of serviced, blocked, and dropped flows.

\section{Acknowledgment}

This study was supported by the "Samsung Electronics Semiconductor Business".

\section{Endnotes}

an the fifth band group, two bands are available for frequency hopping.

${ }^{\mathrm{b}}$ In band group 5, the numbers of the shift are 0,1 only at TFC 8 .

${ }^{c}$ This does not mean that 14 users are the maximum that can be admitted. For instance, the maximum length of BP is 96 beacon slots which support 94 users (two beacon slots are reserved for signaling) in a piconet at maximum. ${ }^{\mathrm{d}}$ In this case, we consider TFC1 and TFC2 in band groups 1, 2, 3, and 4 . eWe have not considered resource allocation for voice traffic because its bandwidth requirement is too small. The allocation of even an MAS is too much for a voice traffic. Note that the SIZ policy cannot allocate one MAS in pieces over three SFs.

Competing interests

The authors declare that they have no competing interests. 


\section{References}

1. J Lee, Y Su, C Shen, Comparative study of wireless protocols: Bluetooth, UWB, ZigBee, and Wi-Fi. in 33rd Annual Conference of the IEEE Industrial Electronics Society (IECON), 46-51 (5-8 November 2007)

2. ECMA-368, High Rate Wideband PHY and MAC Standard. in European Computer Manufacturers Association (ECMA), 3rd edn. New York, 53-76 (3 December 2008)

3. R Zhang, R Ruby, J Pan, L Cai, X Shen, A hybrid reservation/contentionbased MAC for video streaming over wireless networks. IEEE J. Sel. Areas Commun. 28(3), 389-398 (2010)

4. Z Zhang, Y He, E Chong, Opportunistic scheduling for OFDM systems with fairness constraints. EURASIP J. Wireless Commun. Networking. 2008, 1-12 (2008). doi:10.1155/2008/215939

5. O Falowo, H Chan, Adaptive bandwidth management and joint call admission control to enhance system utilization and QoS in heterogeneous wireless networks. EURASIP J. Wireless Commun. Networking. 2007, 1-11 (2007). doi:10.1155/2007/34378

6. F Sun, M You, V Li, Dynamic subcarrier allocation for real-time traffic over multiuser OFDM systems. EURASIP J. Wireless Commun. Networking. 2009, 1-9 (2009). doi:10.1155/2009/298451

7. Y Choi, S Oh, S Choi, S-ARQ: a new truncated ARQ for IP-based wireless network. J. Commun. Networks. 12(2), 174-180 (2010)

8. Part 11, Wireless LAN medium access control (MAC) and physical layer (PHY) specifications. in IEEE 802.11-2007 (12 June 2007)

9. S Kim, K Hur, J Park, D Eom, K Hwang, A fair distributed resource allocation method in UWB wireless PANs with WiMedia MAC. J. Commun. Networks. 11(4), 375-383 (2009)

10. W Kuo, H Wu, Supporting real-time VBR video transport on WiMedia-based wireless personal area networks. IEEE Trans. Vehicular Technol. 58(4), 1965-1971 (2009)

11. Z Fan, Bandwidth allocation in UWB WPANs with ECMA-368 MAC. Elsevier Comput. Commun. 32(5), 954-960 (2009)

12. D Wong, F Chin, M Shajan, Y Chew, in Saturated Throughput of Burst Mode PCA with Hard DRPs in WiMedia MAC (March 2008)

13. R Ruby, J Pan, in Performance Analysis of WiMedia UWB MAC (June 2009)

14. M Daneshi, J Pan, S Ganti, in Towards an Efficient Reservation Algorithm for Distributed Reservation Protocols 1-9 (March 2010)

15. W Alliance, WiMedia Logical Link Control Protocol, draft., 1.0 (13 August 2007)

16. A Khalil, M Crussiere, J Helard, Adaptive self-learning resource allocation scheme for unlicensed users in highrate UWB systems. Springer Wireless Personal Commun 1-13 (2010). doi:10.1007/s11277-010-9993-8

17. A Khalil, M Crussiere, J Helard, in Cross-Layer Resource Allocation Scheme for Multi-Band High Rate UWB Systems (June 2009)

18. S Ko, H Kwon, B Lee, Distributed uplink resource allocation in multi-cell wireless data networks. J. Commun. Networks. 12(5), 449-458 (2010)

19. P Xue, P Gong, D Kim, Enhanced IEEE 802.15.3 MAC protocol for efficient support of multiple simultaneously operating piconets. IEEE Trans. Vehicular Technol. 57(4), 2548-2559 (2008)

20. R Al-Zubi, M Krunz, Interference management and rate adaptation in OFDMbased UWB networks. IEEE Trans. Mobile Comput. 9(9), 1267-1279 (2010)

21. D Lowe, X Huang, in Adaptive Time-Frequency Codes for Ultra-wideband (2007)

22. D Leith, $P$ Clifford, in A Self-Managed Distributed Channel Selection Algorithm for WLANs (April 2006)

23. K Duffy, N O'Connell, A Sapozhnikov, Complexity analysis of a decentralised graph colouring algorithm. Inf. Process. Lett. 107(2), 60-63 (2008). doi:10.1016/j.ipl.2008.01.002

24. M Al-Ayyoub, M Buddhikot, H Gupta, in Self-regulating Spectrum Management: a Case of Fractional Frequency Reuse Patterns in LTE Networks (April 2010)

25. MATLAB http://www.mathworks.com

doi:10.1186/1687-1499-2011-150

Cite this article as: Chang and Bahk: Throughput enhancement using synchronization and three-dimensional resource allocation. EURASIP Journal on Wireless Communications

and Networking 2011 2011:150.

\section{Submit your manuscript to a SpringerOpen ${ }^{\mathcal{O}}$ journal and benefit from:}

- Convenient online submission

- Rigorous peer review

- Immediate publication on acceptance

- Open access: articles freely available online

- High visibility within the field

- Retaining the copyright to your article

Submit your next manuscript at $\gg$ springeropen.com 